AVANT, Vol. XI, No. 2

ISSN: 2082-6710 avant.edu.pl/en

DOI: 10.26913/avant.2020.02.17
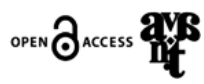

\title{
Understanding the interface between society and cognition
}

\author{
Marcin Trybulec \\ Maria Curie-Skłodowska University in Lublin \\ marcin.trybulec@umcs.pl
}

Received 1 June 2020; accepted 5 August 2020; published 3 October 2020

Keywords: social cognition; situated cognition; group mind; mindreading; cognition as sociomaterial practice; mind as cultural artefact; Daniel C. Dennett

The aim of the special issue "Understanding Social Cognition" is to transfer Aristotelian claim about humans as social beings from the political into the cognitive domain, as well as explore its implications for philosophy of mind and cognitive sciences. Recent decades have made it increasingly evident that cognition is deeply embedded into the collective lives of groups, institutions and societies. Being raised by others, cooperating and coming into conflict with them, inheriting material and institutional environments do not happen by chance but constitute the essential features of the way we are. The Aristotelian dictum that "he who by nature and not by a mere accident is without a state, is either above humanity, or below it" (Politics, 1253a2-4) has been echoed by recent trends in situated cognition, largely inspired by Daniel Dennett, who argues that "Every human mind (...) is a product not just of natural selection but of cultural redesign of enormous proportions" (Dennett, 1996, p. 153). If, as argued by Dennett, human minded behavior is a product of bio-cultural co-evolution, the "machine" for a distinctively human way of thinking is not inscribed in the brain alone but has to be installed in a society that relies on the coordination by embodied agents. This means that socio-cultural dynamics does not just enhance or aid brain-bounded cognition from the outside but cognition itself is intrinsically social. This inference is almost a platitude among the social scientists, since they agree that groups exhibit dynamics which cannot be reductively explained or predicted solely by reference to actions and properties of their individual members. Classic research in social psychology delivers convincing evidence to support this claim. For instance, the structure of a working group and its patterns of communication can substantially improve the efficiency and reliability of problem solving. This can be observed even in small, fiveperson groups. Such collectives with centralized communication patterns perform consistently better than decentralized groups, when confronted with a simple task (Bavelas, 1948). In this kind of tasks, the structural properties of the group act as an amplifier, enabling the collective 
to overcome the cognitive limitations of its individual members. While these findings inspired new insights in sociology and organization studies in the $20^{\text {th }}$ century, philosophy and cognitive science have been slow to embrace the idea that group level dynamics is a crucial factor in explaining mind and cognition. Moreover, the claim that the mind and cognition might be essentially social seems to be at odds with much of philosophy and cognitive science.

Contemporary research on the distinctively social aspects of human cognition, which has been flourishing in the last two decades, tends to make up for this oversight. New conceptual and empirical investigations cover the whole gamut of social cognition: from elementary embodied behavioral coupling and joint intentionality, through social-cognitive skills that allow coordination and mutual understanding, all the way up to group cognition. These considerations can be divided along ontological and epistemological lines. First, in the domain of social ontology, the question is what kinds of minds there are. Can a group display a mind of its own? The discussion falls somewhere along the continuum between the "conservative" and the "liberal" take on the ontological status of the group mind and group cognition. On the "conservative" side, the minds of individuals are reconceived as socially situated, culturally scaffolded, and substantially transformed by life-long immersion in social life, but the very idea of the group mind is still questioned (Rupert, 2011). According to more "liberal" multi-level approaches, the informational integration of functionally interdependent and socially distributed individual cognitive processes can constitute emergent group-level phenomena, such as "mental commons" or "plural subjects" (Gilbert, 2004; Theiner, Allen \& Goldstone, 2010).

The questions about the ontological status of a group agent or group cognition are rooted in more basic issues regarding the processes of mutual understanding, which occur at the level of two individuals. Mindreading processes can spark the emergence of the group level cognitive phenomena by enabling new ways of organizing cognitive work. Only those who manage to recognize the intentions of others are able to engage in specifically human ways of learning, make sense of the material tools and appreciate institutional practices, inherited from past generations, therefore participating in communal effort, called human cognition. That is why the question of how people make sense of themselves, others, and their environment should not be limited to the narrow domain of psychological research, but is essential for anyone concerned with cognition at large (Tomasello, 2018). This leads to another set of questions in the domain of social cognition: How do we know about other minds and what is the mechanics of mindreading?

Independently of the issue of mindreading, one shall ask the question of how an individual fits into the broader collective dynamics. Integrating philosophical and psychological accounts of social cognition into the wider framework of distributed, situated and enactive cognition brings us closer to answering the question of how exactly individuals depend on one another and their epistemic environments in performing cognitive work (Hollan, Hutchins \& Kirsh, 2000). If, as argued by Dennett (2017), mind is a type of cultural artifact, the processes of cultural transmission, sharing information and picking up opportunities embedded in the environment gain central position in the debate about thinking and rationality. 
The papers contained in this issue explore the question of how cognitive processes displayed at the level of an individual are linked with cognition conceived as collective effort, situated in material and cultural contexts. This volume is divided into four sections according to the issues lying at the heart of the domain of social cognition. The first section focuses on the fundamental questions about the epistemological and ontological nature of group agency, whether cognitive actions observed at the level of collectives belong to the same natural kind as cognitive processing at the level of an individual, and whether the idea of collective agency is something more than just instrumental abstraction used for predicting the group performance. The second section is concerned with conceptualizing the processes of mutual understanding, how to make sense of social cognition beyond representational framework, what the appropriate level of abstraction is needed for explaining the mechanism of social cognition and what the role of interaction and social practices is in gaining skills in understanding oneself and others. The third section reconsiders cognition and consciousness as deeply socially situated, raising questions of how far one can go in explaining cognition without referring to intracranial representational processes and how to describe thinking and decision-making in terms of distributed dynamics of socio-material practices. The last section is devoted to Daniel C. Dennett's book From Bacteria to Bach and Back. The Evolution of Minds (2017), where the contributors struggle to scrutinize basic ideas used by Dennett to explain how it was possible that mindless processes of natural selection gave rise to mindful behavior of modern humans and how to make sense of cultural transmission in naturalistic evolutionary framework. In responses to challenges raised toward some aspects of his approach D.C. Dennett reveals his intuitions and assumptions lying behind his understanding of information, communication, memes and affordances.

\section{Understanding group mind}

The first contribution to the special issue, "Group minds and natural kinds" (2019), addresses the question of how to decide whether cognition considered at the level of an individual and at the level of groups are the instantiations of the same natural kind. Robert Rupert wonders what kind of evidence can support realism about group cognition. In order to tackle this problem, the author presents an abstract model of the cognitive system and asks if this model supports the hypothesis that groups can have cognitive states of their own. Particularly, he raises concerns whether extremely specialized and inflexible collective intelligence can be fitted into the same natural kind as all-purpose intelligence, displayed by individuals. Moreover, Rupert indicates that the whole set of methodological principles, such as simplicity of explanations, cast doubt on the existence of genuine group-level cognitive states. Finally, he concludes that the current theory as well as the available data do not support the idea that group cognitive states, such as memory or belief can be understood in the same way as the cognition of individuals.

The issue of group agency is also considered by Sven Delarivière (2020), who, in "Collective understanding", employs a Dennett-style argumentation that recognition of group-level epistemic agency depends on the particular way of looking at the phenomena in question. He proposes that in order to make sense of group agency, one needs to apply an "epistemic stance" - 
an idea modeled on Dennett's "intentional stance". Delarivièrere constructs two thought experiments to showcase when it would be required to adopt an "epistemic stance" toward a group of individuals. By examining arguments regarding complexity, macro-systematicity and emergence, the paper explores the conditions for attributing epistemic agency to a group. On this view, the idea of group agency is a useful instrument applied in order to predict or evaluate the group's performance.

\section{Understanding other minds}

The paper "Symbolically Mediated Interaction and Perspective-Taking" by Duygu Uygun Tunc (2019) puts emphasis on the mechanisms of social interaction as an essential factor in explaining perspective taking. Tunc identify the blind spots in competing theories of social cognition; in traditional representational theories and in the embodied approach. She questions the tendency to frame representation and interaction as mutually exclusive, which is observed in both research traditions. Instead of siding with one of the approaches, the author looks for a middle-ground. Drawing on the symbolic interactionism of G. H. Mead and L. S. Vygotky, the paper explores the question of how the ability of perspective-taking develops. Tunc embraces the fact that practices of perspective-taking are already immersed in a reach environment of symbolic routines. At the early stages of development, however, those symbolic structures are external to an individual. According to symbolic interactionism, children internalize symbolic structures implicit in social environment by simply enacting the patterns of interactions. Therefore, they are gradually gaining skills in mental construal of perspectives, which opens up new dimensions of social cognition.

In "Relations between self-understanding and other-understanding", Adrianna Smurzyńska (2020) examines the correlations between self- and other-understanding in the leading theories of mentalization: theory theory, simulation theory and person model theory. After presenting the main approaches to social cognition and sketching how each approach conceptualizes the relations between self- and other-understanding, the author is inclined to maintain that although the processes of self- and other-understanding are closely interdependent, they stem from different mechanisms. Smurzyńska emphasises that both self- and other-understanding involve attribution of the same types of mental states, as well as interpretation of the same types of behavioral cues. Additionally, both abilities operate upon imaginative and reflective thinking. According to the author, the most relevant differences between the ability to understand the self and the others hinge upon the nature of phenomenological experience of agency and, in some cases, self-understanding involves a higher level of attribution accuracy.

\section{Understanding cognition as an embodied-social practice}

“Social and Enactive Perspectives on Pretending” by Zuzanna Rucińska (2019) presents an analysis of how social practices and interactions might be instrumental in explaining children's abilities to engage in pretend play. This is due to the fact that pretense itself is constituted by these practices and interactions. She demonstrates that in explaining pretense, the mainstream approaches assume imaginative and representational processes as a solution to what she calls, 
a 'bypassing challenge'. Rucińska develops an argument that the problem of sidestepping the most obvious response to the environment does not exist simply because there is no obvious response that needs sidestepping. In pretense there is no default interpretation of an object, and people simply respond to different, playful affordances of an object with which they interact. Since meaning of the object is created in action and interaction with others, invoking cognitive architectures to replace one representation for another is unnecessary for explaining pretense. The author shows how the enactive account of social cognition can be applied in order to deliver an alternative explanation of pretense. By framing pretense as intrinsically social a process enabled by direct perception of socially constructed affordances and the history of patterned interactions with the caregiver - she shows that explanation of pretending does not need to assume special cognitive architectures, including specialized internal scripts. In its final parts, the paper presents a sketch of the mechanisms for perspective taking, imitation, affect and perception that can provide explanations of the pretend play.

In "Neural-based vs. Enactive Approaches to Consciousness and Social Cognition", Zsuzsanna Kondor (2020) reviews ideas regarding the relationship between cognition and selfconsciousness, developed in phenomenologically inspired embodied cognitive science. The author starts with surveying the attempts at conceptualizing cognition and consciousness in traditional philosophy of mind and contrasts it with approaches inspired by M. Marleau-Ponty and L. Vygotsky. Kondor employs the "social brain hypothesis" in order to demonstrate the continuity between the individual and social dimensions of cognition and consciousness, considered on the evolutionary time-scale. The paper explores the possibility that the focus on the social dimension of mind in enactivist approaches might be instrumental in building bridges between consciousness and cognition.

In "Writing as distributed socio-material practice", Aleksandra Kołtun (2020) seriously approaches the claim that cognition and knowledge can be collectively constructed by groups of people through the skillful use of external symbolizations. By delivering a detailed ethnographic description of how civil servants and citizens cooperate in creating regulations for participatory budgeting, she builds an empirically supported case for the claim that human beings are social organisms who make sense of each other and the external world through technologically and socially situated practices. She describes how in a complex writing task, thinking is distributed across time and space and coordinated between human agents or material artifacts such as flip-charts, hand-notes and successive printed versions of documents. Kołtun's account of the distributed dynamics of socio-material practice of writing can be conceived as an argument against the organism-centered view of thinking.

\section{Understanding the evolution of minds by Daniel C. Dennett}

“Thinking about Semantic Information” by Marcin Miłkowski (2020) initiates a set of critical appraisals of Dennett's perspective on the bio-cultural transmission of information. Miłkowski casts doubt on Dennet's account of semantic information as non-measurable and independent of encoding. In his contribution, Miłkowski emphasizes that any explanation regarding semantic information has to cope with the question of how information with semantic content is rooted in non-semantic information. At the same time, respectable theories of non-semantic 
information frame information in terms of encoding and aim at measuring it. Therefore, in order to transfer semantic information to any receiver a description of the encoding procedure is necessary. Dennett designed a thought experiment intended to show that in some situations, one can share semantic information without encoding. In response, Miłkowski argues that his thought experiment can only support the claim that it is actually possible to share the same semantic information with various types of encoding. Therefore, Dennett's argument by itself cannot stand in support for the claim that sharing a piece of information is possible without encoding it. According to Miłkowski, no conceptually and scientifically acceptable theory of semantic information can proceed without due attention to how the information is encoded and measured.

Dennett's (2020a) sympathetically responds to attempts at clarifying the concepts of semantic information, encoding and measurement. He casts doubt however on Miłkowski's optimism about bridging the conceptual gap between formal take on semantic information and theories aiming at describing information embodied and sheared by real world agents. Dennett puts forth conceptual and empirical cases of cultural transfer, linguistic creativity and indeterminacy of meaning to showcase the role of socially shared background knowledge which - despite huge diversity of codes - enables human beings to reach mutual understanding. In this way Dennett justifies his skepticism toward the prospects of drawing together sematic and non-semantic information by appealing to the ideas of coding and measurement.

Zuzanna Rucińska's commentary, “Affordances in Dennett's From Bacteria to Bach” (2020), introduces the question of what explanatory role affordances play in Dennett's evolutionary theory of cognition. She traces the conceptual obstacles on the way towards a fruitful integration of affordances within Dennett's approach. The first issue is generated by the fact that there is no commonly accepted ontology of affordances and the meaning of the term varies from theory to theory. Rucińska points out that without a clear ontology of affordances, it may be difficult for them to play a useful, scientifically respectable role in cognitive science. She showcases what kinds of interpretations of affordances could be chosen, pointing out that they cannot all 'go together', because they are inconsistent. Struggling to find the most fruitful way of conceptualizing affordances, she identifies a second issue, concerned with the epistemic access to it. If conceptual relations between affordances, ecological information and semantic information are not properly defined, the question arises whether affordances are picked up in the same way as information is. In addition, the author suggests that it is unclear whether affordances are picked up or created, as it is the case in the socio-cultural domain. The question of what is responsible for recognizing affordances introduces the third problem with Dennett's perspective. Rucińska casts doubt on the claim that the brain alone can be responsible for recognizing opportunities delivered by human socio-cultural cognitive niche. She concludes that if affordances are to be instrumental in cognitive explanations, their ontological status as well as the epistemic access to it need to be carefully scrutinized.

In his response to Rucińska, Dennett (2020b) agrees that from the philosophical point of view the concept of affordance needs a clarification. At the same time he argues that philosophical puzzles, such as debates about ontological status of colors or metaphysical nature of affordances, usually have little relevance for scientific research which can flourish regardless of 
the multiple understanding and sometimes inconsistent definitions of the phenomena in question. Although Dennett is not convinced that conceptual challenges raised by Rucińska are serious enough to be picked up by empirical researchers, he encourages her to develop the conceptualization of affordances which might lead to interesting insights into their structure and functions.

In "Can memes explain the birth of comprehension", Paweł Grabarczyk (2019) examines the role of memes in Dennett's ideas about the origins of a distinctively human form of intelligence. In this way, he introduces the fundamental problem of how comprehension emerged from non-reflective and automatic competence. While he accepts the conceptual distinction between competence and comprehension, he denies that memes can be used as an explanatory tool in enlightening the transition from the former to the latter. Grabarczyk identifies two interconnected reasons for that. First, he argues that the paradigmatic examples of memes (i.e. words) imply the notion of linguistic meaning, which itself requires explanation. Second, Grabarczyk explains why without a thorough examination of the idea of meaning, Dennett's approach is ambiguous as regards the identity conditions for the replication of memes, and this problem is pivotal in explaining the mechanism of their cultural transfer. In consequence, Dennett's use of memes as an explanatory tool does not succeed in bridging the gap between competence and comprehension. Grabarczyk concludes by pointing towards a more coherent view on cognitive evolution, which, in his opinion, is possible by combining Brandom's normative account of language with Dennett's evolutionary approach.

Dennett (2020c) in line with Grabarczyk acknowledges that normativity shall be involved into explanations concerning linguistic comprehension or cultural transmission. He sketches distinction between higher order social norms (a concept applied by Brandom) and more basic instrumental normativity conceived as means/ends coherence. Dennett sides with Hume in maintaining that the kind of normativity applied by Grabarczyk after Brandom should be grounded in more basic utilitarian idea of a norm. While considering the problem of identity conditions for meme's replication Dennett points out that by virtue of socially shared background knowledge the transfer of memes can proceed in the absence of clear-cut criteria or definitions. By appealing to the process of acquiring cultural skills he makes a case for the claim that meme's propagation allow their more or less implicit understanding. Finally, Dennett by appealing to the Socratic ideal of continuous self-interpretion responds to Grabarczyk's skepticism about the quest for understanding how comprehension emerge out of mere competence.

\section{Acknowledgments}

The papers enclosed in this special issue are the products of the third edition of "Trends in Interdisciplinary Studies", the conference held in Lublin, on October 20-22, 2017. The conference gathered researchers of diverse disciplinary backgrounds, from philosophy, psychology and sociology to linguistics and cognitive science providing a rich source of perspectives, research, arguments and insights for those who are interested in social cognition, in a broad sense. The conference organizers owe a debt of gratitude to Maria Curie-Skłodowska Univer- 
sity, John Paul II Catholic University of Lublin and the Ministry of Science and Higher Education (grant no: 762/P-DUN/2017) for providing generous support, necessary to organize the event. We particularly wish to thank Akadiusz Gut, Andrzej Łukasik, and Witold Wachowski for their support and trust that the project will succeed. We are also grateful to Karol Jałochowski, Marcin Miłkowski and Georg Theiner for their valuable advice and guidance. Special thanks go to, Aleksandra Kołtun, Robert Mirski, Marcin Rządeczka and Marcin Zaród, whose engagement and effort made the event possible.

\section{Funding}

The work on the special issue and the paper were supported by National Science Center, Poland, under the grant Understanding Cognitive Artifacts: Towards the Epistemology of Cognitive Extensions, no. 2017/26/D/HS1/00677.

\section{References}

Aristotle. (1885). Politics, (transl. M. A. Jowett). Oxford: Clarendon Press.

Bavelas, A. (1948). A mathematical model for group structures. Applied Anthropology, 7(3), 1630. doi: 10.17730/humo.7.3.f4033344851g1053

Delarivière, S. (2020). Collective Understanding-A conceptual defense for when groups should be regarded as epistemic agents with understanding. Avant, 11(2). doi: 10.26913/avant.2020.02.01

Dennett, D. C. (1996). Kinds of minds: Toward an understanding of consciousness. New York: Basic Books.

Dennett, D. C. (2017). From bacteria to Bach and back: The evolution of minds. New York: WW Norton \& Company.

Dennett, D. C. (2020a). Comment on "Thinking about Semantic Information." Avant, 11(2). doi: 10.26913/avant.2020.02.15

Dennett, D. C. (2020b). Comment on "Affordances in "Dennett's 'From Bacteria to Bach and Back'." Avant, 11(2). doi: 10.26913/avant.2020.02.14

Dennett, D. C. (2020c). Comment on "Can memes explain the birth of comprehension?." Avant, 11(2). doi: 10.26913/avant.2020.02.13

Gilbert, M. (2004). Collective epistemology. Episteme, 1(2), 95-107. doi: 10.3366/epi.2004.1.2.95

Grabarczyk, P. (2019). Can memes explain the birth of comprehension? Avant, 10(3). doi: 10.26913/avant.2019.03.29

Hollan, J., Hutchins, E., \& Kirsh, D. (2000). Distributed cognition: Toward a new foundation for human-computer interaction research. ACM Transactions on Computer-Human Interaction, 7(2), 174-196. doi: 10.1145/353485.353487

Kołtun, A. (2020). Writing as distributed sociomaterial practice - a case study. Avant, 11(2). doi: 10.26913/avant.2020.02.16 
Kondor, Z. (2020). Neural-based vs. Enactive Approaches to Consciousness and Social Cognition. Avant, 11(2). doi: 10.26913/avant.2020.02.02

Miłkowski. M. (2020). Thinking about Semantic Information. Avant, 11(2). doi: 10.26913/avant.2020.02.08

Rucińska, Z. (2019). Social and Enactive Perspectives on Pretending. Avant, 10(3). doi: 10.26913/avant.2019.03.15

Rucińska, Z. (2020). Affordances in Dennett's From Bacteria to Bach and Back. Avant, 11(2). doi: 10.26913/avant.2020.02.05

Rupert, R. D. (2011). Empirical Arguments for Group Minds: A Critical Appraisal. Philosophy Compass, 6(9), 630-639. doi: 10.1111/j.1747-9991.2011.00420.x

Rupert, R. D. (2019). Group Minds and Natural Kinds. Avant, 10(3). doi: 10.26913/avant.2019.03.08

Smurzyńska, A. (2020). Relations between self-understanding and other-understanding: similarities and interactions. Avant, 11(2). doi: 10.26913/avant.2020.02.07

Theiner, G., Allen, C., \& Goldstone, R. L. (2010). Recognizing group cognition. Cognitive Systems Research, 11(4), 378-395. doi: 10.1016/j.cogsys.2010.07.002

Tomasello, M. (2018). A natural history of human thinking. Cambridge, MA: Harvard University Press.

Tunc, D. U. (2019). Symbolically Mediated Interaction and Perspective-taking: A Social-relational Perspective on Social Cognitive Development. Avant, 10(3). doi: 10.26913/avant.2019.03.28 